\title{
FRAMEWORK ARCHITECTURE FOR IMPROVING HEALTHCARE INFORMATION SYSTEMS USING AgENT TECHNOLOGY
}

\author{
Hasan Omar Al-Sakran \\ Management Information Systems Department, King Saud University, Riyadh, Saudi \\ Arabia
}

\begin{abstract}
Due to diversity, heterogeneity and complexity of the existing healthcare structure, providing suitable healthcare services is a complicated process. This work describes the conceptual design of an e-healthcare system, which implements integration strategies and suitable technologies that will handle the interoperability problem among its essential components. The proposed solution combines intelligent agent technology and case based reasoning for highly distributed applications in healthcare environment. Intelligent agents play a critical role in providing correct information for diagnostic, treatment, etc. They work on behalf of human agents taking care of routine tasks, thus increasing speed and reliability of the information exchanges. CBR is used to generate advices to a certain e-healthcare problems by analyzing solutions given to previously solved problems and to build intelligent systems for disease diagnostics and prognosis. Preliminary experimental simulation based on Agent Development Framework (JADE) demonstrated the feasibility of this model.
\end{abstract}

\section{KEYWORDS}

Multi-Agent Systems, Interoperability, Case Based Reasoning

\section{INTRODUCTION}

Most of the healthcare information systems are developed by the different vendors and built on different multi-platforms (different system architecture, infrastructure and databases). Most of the healthcare software development organizations work in island mode which leads to complications with regard to integrity and interoperability among different healthcare systems. Interoperability is the ability of different subsystems to access and to use the data reliably and quickly from various sources without the occurrence of errors [1]. The interoperability among the healthcare information systems enables these systems to communicate with each other in order to share information, improving its high availability [2].

Healthcare information systems lack standardization, so sharing and integrating information in such systems is quite difficult. Consequently, providing suitable healthcare services is complicated due to the diversity, heterogeneity, spreading out over multiple locations, and complexity of these healthcare systems. Maintaining software from various sources and associated interfaces are very costly operations.

In the current healthcare scenario, patients have multiple health records in different healthcare information systems, which mean patient-related information being fragmented into different systems. The need to access patient data across these systems and manage the information flow between various systems is adding to the complexity of healthcare information systems. 
Therefore, interoperability in healthcare information systems ever more becomes a requirement rather than a feature.

Healthcare system can be characterized by presence of a large number of actors, such as healthcare professionals (physicians, nurses, pharmacists, administrators, etc), a large number of services (outpatient care services, rehabilitation services, etc.), and highly specialized professionals. In order to improve the quality of treatment and ensure more accurate diagnostics, the activities of these actors need to be coordinated and controlled using efficient communication techniques. Even in sophisticated healthcare information systems, such as e-Health, specialist referral services, patient monitoring, e-prescriptions, e-medical records, Hospital Management Information System, Telemedicine Information System, Laboratory Information System, etc., the information flow still requires interaction between actors either face-to-face or via a communication system where users must be physically present at either end of the communication link to successfully establish a synchronous interaction.

Healthcare systems, in general, face unique challenges that include inadequate infrastructure, little or no access to continuous healthcare education, limited access to primary healthcare facilities, and large distances from advanced care centers. Many healthcare information systems assume that users are working on fixed workstations at fixed locations, not taking into consideration the latest advances in mobile technology such as mobile phones and smart mobile devices. Therefore, healthcare information system, in general, requires reengineering of their service processes.

The primary motivation of this work is to develop a comprehensive solution to the integration and interoperability problems within healthcare information systems. This work focuses on conceptual design of a healthcare information system. We propose an efficient agent-based architecture of healthcare system supported by CBR. Combination of agent and CBR technologies offers an effective solution for the above mentioned problems in terms of better integration, which, in turn, results in more efficient access to different healthcare systems, and allows acquisition of patient's medical data (such as radiological and laboratory results) and monitoring information from multiple sources, thus improving patient's care, including diagnostics and treatment. Distributed Electronic Patient Records (EPR) allow medical staff to gain access to patient's medical information, such as test results, clinical data, etc., independent of applications they are using or of data locations, at convenient time, online or offline.

The proposed solution takes into consideration the concerns of the healthcare administration which are focused on information technology as a means to cut costs and improve quality of healthcare. It is cost-effective in terms of using the existing information and communication technologies for support of health and health-related fields, such as healthcare services, health observation, health literature and health education, and may improve healthcare locally, regionally and worldwide. Such an approach preserves the investment in existing sophisticated ehealth information systems, allows developers to add new features satisfying the needs of growing user mobility, and provides intelligent assistance to users.

Other objectives of proposed framework architecture are to provide access to healthcare services and health information at remote primary healthcare centers as well as within the same center, to help healthcare actors to interact efficiently among themselves (by transferring routine tasks from humans to software agent) and within the information systems. Access to distributed healthcare systems will enable medical staff to gain access to a service that can enable collaboration between different healthcare actors. Such access should be available from anywhere in and around the healthcare organization and will improve the efficiency in medical information acquisition. 
By nature healthcare information is mobile, since patient's information is needed by medical professionals at different locations to improve diagnostic, efficient care, and reduce medical errors. Timely information in critical situations can make a difference between life and death. Applying wireless computing in healthcare environment will improve the quality and reduce the cost of healthcare. However, the large number of patients and their related data cannot be efficiently processed by the current systems, so an efficient processing approach is needed for real-time response requirements for critical health situations. In the proposed system we use wired and wireless communications to connect patients, doctors and hospitals staff into a unified healthcare system. Modern mobile communications provide wide coverage, low cost, high efficiency, reliability and availability of services. Mobile devices in e-healthcare can be used to collect, transmit and process vital patients' data, for example, heart rate and blood pressure, in real time. Such systems are especially important in applications that remotely monitor patients with chronic ailments or in homecare.

The rest of the paper is organized as following. Background on agent and CBR technologies are discussed in section 2. Related work is presented in section 3. Section 4 will introduce the overall framework of the proposed healthcare information system. Discussion of the proposed system is presented in section 5. Finally, section 6 is devoted to conclusions and future work.

\section{TECHNOLOGY OVERVIEW}

The most suitable way to handle the interoperability problem among the essential healthcare components in distributed healthcare environment is combination of intelligent agents and case based reasoning. The distinctive features of each, beneficial for the proposed system, are described below.

\section{1 Agent Technology}

In the client-server model communicating entities have fixed and well defined roles. A server offers a set of services and a client makes use of these services. They communicate through a message passing protocol. But message passing requires programmers to determine network addresses and synchronization points.

In the mobile agent model, when a client agent requires access to a resource provided by server, it moves to the server site and makes requests to the server directly. When the transaction is complete, the mobile agent returns back to its original site with the results.

Mobile agents are reusable intelligent executable software, capable of supporting lightweight computations and carrying out autonomous tasks. Agent has the ability to predict the needs of users and adjust to these needs. It can learn new techniques and make suggestions to a user. The learning process is derived from a user's response and an assessment of previous performance. An agent has a degree of control over its actions and it does not have always to wait for commands. Agents can coordinate their actions, cooperate with other agents and act on their own initiative to achieve their goals and adapt their behavior in response to the changing environment. An agent can migrate from server to server in heterogeneous networks. On each server, the agent interacts with stationary services and other resources to accomplish its mission. It can communicate to anticipate, adopt and plan tasks. Its behavior consists of beliefs, desires, and interaction depending on the place function of an entity within an agent-based system. 
A mobile agent consists of two different parts: the code itself, which composes of the instructions that define the behavior of the agent and its intelligence, and the current state of execution of the agent.

Agents use a platform for developing agent applications and to operate so as to achieve their objectives. A lot of platforms are available: JADE [3], JACK [4], AgentBuilder [5], Aglet [6], D'Agents, Fargo, Voyager, etc., to name a few. JADE (Java Agent Development Environment) is a Java-based open source development framework aimed at developing multi-agent systems and applications. JADE-LEAP (Java Agent Development Framework-Lightweight Extensible Agent Platform), the active agent platform on all mobile devices, is an agent-based runtime environment that is targeted towards resource constrained mobile embedded devices.

The agent platform JADE and JADE-LEAP support the following tasks: creating, starting, deleting and migrating of agents; agent communication, where the executing environment is used to pass messages between agents; agent administration and errors announcement.

To construct a mobile agent, there are at least three major requirements such as:

- Common execution language across heterogeneous networks, for example Java (Due to its strengths: portability, mobility, and server security).

- Mobility of agents across networks through a communication mechanism, for example MAP, TCP/IP, HTTP, or SMTP, or other transport mechanisms.

- Protection of agents against hostile server and agent server from malicious agent. Agent may protect their data and information through encryption/decryption techniques.

The choice of the agent language should be compatible with the requirements needed in a healthcare setting. It's composed of a common protocol, a common interchange format, and is adaptable to changes in the overall e-health environment (its actors, the content, medical services and hardware resources available).

Agent communication can be conducted using two methods: synchronous and asynchronous. In the first method the communicating agents must arrange for a time to communicate and must synchronize before data can be transferred. This method is used for situations where data transfer is important and needs to be confirmed. In the second method collaborating agents will communicate with each other when the receiving agent checks for received data.

\subsection{Case-Based Reasoning (CBR) Methodology}

CBR is a methodology in which knowledge is derived directly from historical cases. CBR is a well established methodology for construction healthcare systems [7, 8]. One of the attractive features of CBR in medicine is that the concepts of diseases can be naturally represented as cases. Case can be obtained from diagnosing a particular patient with a particular disease. Every day practice is collected and stored as cases. Therefore, the medical information system has a rich collection of past problem-solving cases stored together with their solutions. Reasoning with cases is similar to the decision making process of physicians. Physicians are comfortable using CBR methodology in dynamically changing situations were much is unknown and when diagnoses are not clear.

CBR is used to generate solutions to a certain e-healthcare problem by analyzing solutions of previously solved problems. CBR plays an important role in building intelligent systems for disease diagnosis and prognosis. It is becoming fundamental tool for the health sciences [9]. 
There are different medical applications using CBR for diagnosis of heart disorders, for renal function monitoring system, liver transplant patient [10,11], etc.

The CBR approach makes use of previous experience to solve newly encountered problems. Figure 1 shows the principle idea how CBR works.

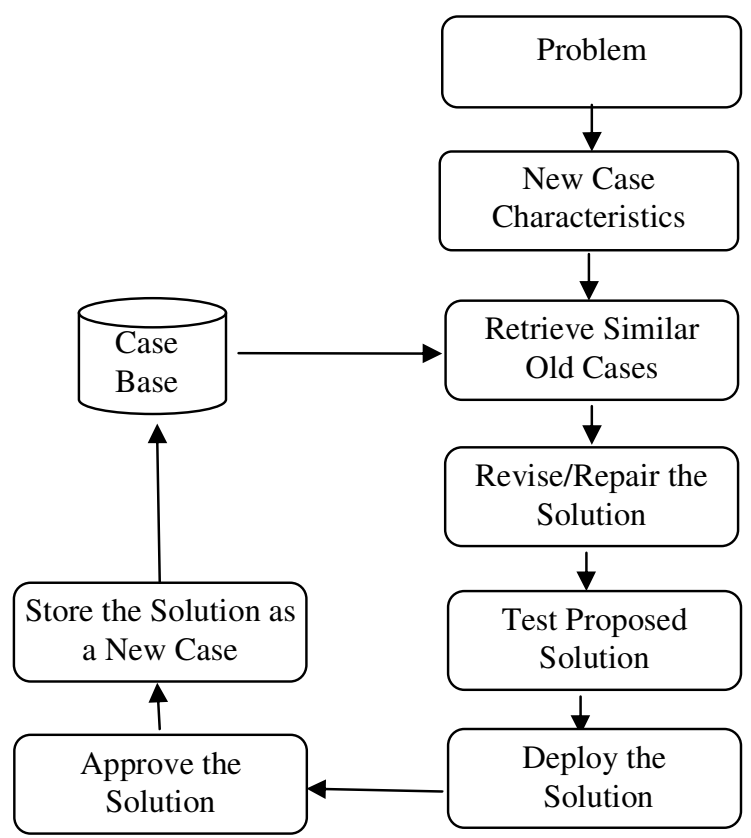

Figure 1. Case Based Reasoning Module

CBR method is based on re-use of the past experience that is organized in the form of cases. The purpose of CBR is to help its user to find and retrieve cases similar to the current problem and adapt them so they can be used to solve this new problem. After achieving the final solution this new medical knowledge can be added to the case base as another case thus expanding the medical knowledge base.

\section{RELATED WORK}

The intention of this research is to integrate all the existing medical subsystems by providing an effective distributed decision support system using mobile agents as a mediator. Several researchers have explored the use of intelligent agents in the medical field $[6-7,12]$. The reason for such an interest is that medical systems are becoming more distributed and consist of complex arrangement of multiple individual systems. The efficient management of such distributed environment becomes very important. There have been many propositions for managing distributed systems. However, one promising piece of technology is the use of mobile intelligent agents.

Different information technologies have been used to accommodate integration of all existing medical subsystems into an effective distributed support system. First efforts for integration of healthcare systems were deployed in standardization. One solution for exchange of medical data was offered by HL7 (Health Level Seven) [13]. HL7 provides standards for exchange, management and integration of healthcare data. Its main objective is to simplify interoperability among heterogeneous healthcare applications. It defines formats for data exchange presented in different healthcare applications. HL7 contains a set of specifications that define how a software 
package compliant with HL7 is implemented and used, and includes a set of message-based transactions between healthcare applications. But in practice the integration at the standards level is not sufficient. Some researchers attempted to achieve interoperability of healthcare information systems using service oriented architectures (SOA) architecture. SOA-based HIS services architecture has been proposed in [14 - 17]. Others [18, 19] proposed to use multiple agent based architecture in conjunction with the HL7 standard as means of interoperability implementation in healthcare systems.

There has been a growing interest to application of agent-based systems in healthcare [12, 20, 21, 23-27] because of the increasing size and complexity of medical systems and urgent need of their efficient management in distributed environment. Zheng and Li [22] proposed a framework to integrate medical information resources using agent and XML. Other researchers apply agent technology for specific medical applications such as monitoring pregnant women with gestational diabetes mellitus [28] and spread of influenza virus infection [29].

Annicchiarico et al. [30] described services that can benefit from introduction of multi-agent systems such as geriatric, diagnostic and telemonitoring e-services, etc. These e-health applications share notable features: loosely coupled heterogeneous systems, dynamic management of distributed resources, and accessibility by remote e-health actors, which corresponds with the characteristics of software agents [30, 31]. Multi-agent systems can be used for the recognition of diagnosis applications and Health Agents IHKA [32], OHDS [33-35]. Lee et al. [36] and Kifor et al. [37] proposed intelligent agent-based healthcare models.

De Meo et al. [38] recommended a multi-agent system to support patients searching for healthcare system that is HL7-aware and detects healthcare service providers delivering requested e-healthcare services. Su and Chu [39] addressed the issues of interoperability and scalability in heterogeneous e-health environments through the adoption of a FIPA2000 standard using agent technology.

\section{AgENT-BASED HEALTHCARE INFORMATION SySTEM}

The agent technology seems an attractive methodology for integrating and developing distributed e-healthcare information systems because it solves the problem of heterogeneity, supports intelligent and distributed storage, allows an optimal personalized e-health environment, and enhances modularity, reusability, flexibility and reliability. Agent may operate as a part of a community of cooperatively distributed system environments and ensure interoperability between different systems that are to be integrated into an operational heterogeneous e-health system. Besides that, it solves the problem of low-bandwidth, reduces network traffic and process data locally instead of transmitting the data over a network. It could accelerate system development by using agent components; and enhance modularity, reusability, flexibility and reliability. Other agent's features to include are cooperation, coordination and interaction as well as intelligence.

Multi-agent systems can become a key part of the e-health applications such as: quality aging eservices helping elderly people, diagnostic, telemonitoring, smart hospital and smart emergency applications. These e-health applications can surely benefit from deployment of agent technology $[30,31]$.

The advantages of using agent solution in healthcare information system:

- No changes to the features and functionalities of current health information systems are necessary;

- Routine and many non-routine tasks will be completed by software agents;

- Actors may receive and transfer data anywhere and anytime through their mobile devices; 
- All transactions are systematically saved to maintain audibility of the system.

\section{1 Proposed Architecture}

This research proposes a hybrid approach combining Case Based Reasoning and multi-agent technology to tackle the above mentioned problems. It's a cooperative distributed solution which allows the numerous healthcare actors to share their information and benefit from sub-systems' capabilities in open distributed healthcare environments. CBR can be successfully used to generate solutions to a certain problem by analyzing solutions given to previous solved problems. A group of intelligent agents will work towards finding an acceptable solution to a problem at hand. Such e- healthcare system will accept user's preferences and pass them to the CBR system where the best matching case or cases are retrieved from a case base. System architecture is shown on Figure 2.

The proposed e-healthcare system will implement different agent categories: intelligent (problemsolving agents), interaction (Interface Agent), and communication (Mobile Agent).

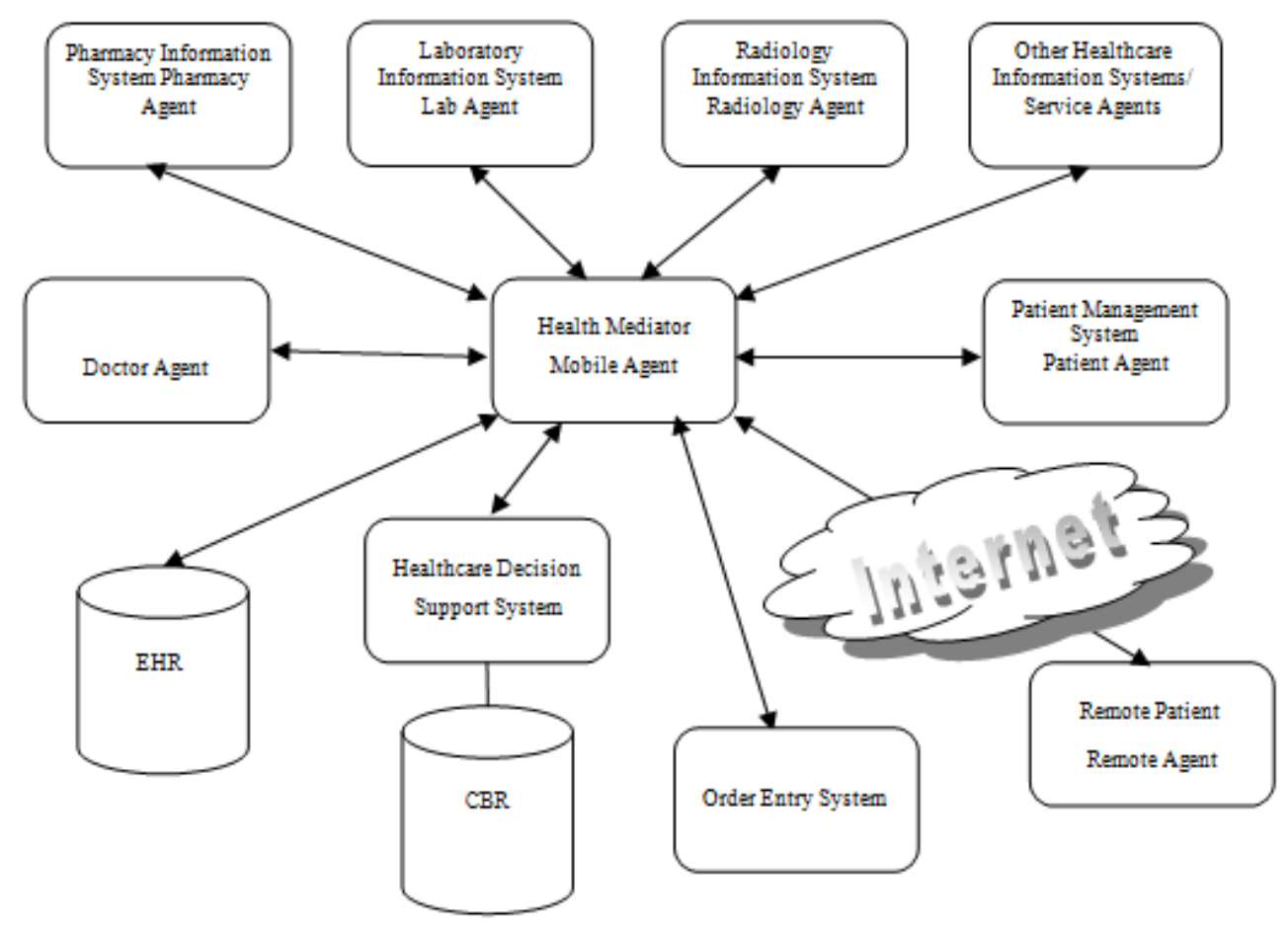

Figure 2. e-Healthcare System Architecture

\section{2 Types of Agents}

The following types of agent are used in the proposed system:

- Interface agent

- Doctor agent

- Prescription agent

- Mobile agent 
- Lab agent

- Diagnostic Agent

- Mobile device agent

- Home patient agent

- Schedule agent

- Electronic Health Record (HER) agent

Each agent consists of its knowledge base and set of rules. An agent might play different roles, assume different responsibilities, and be able to coordinate cooperatively or competitively with other agents. Agent will be assigned to healthcare actors, specify tasks and determine the behaviours of each agent according to their roles in the e-healthcare. Agents' behaviour follows rules based on the requirements of their owners. The responsibilities of each agent are outlined as follows:

\section{Interface Agent (IA):}

The interface agents provide user-friendly interactive interface to assist the user in accomplishing necessary tasks, like filtering retrieved information from queries, etc. It is responsible for sending user requests to the appropriate agents, returning feedback to the user, and final presentation of results generated by other agents. This agent has the capability of learning from user's behavior, feedback and instructions, and from other agents. Karpagam and Saradha [40] proposed to develop conversation interfaces between a system components and human using agents to improve knowledge in the domain of healthcare.

\section{Doctor Agent (DA):}

The doctor agent communicates with other agents to find out which is the most adequate agent to perform the requested service. The main tasks of the agent are:

- Receive report on test results from the lab agents;

- Receive alerts on unread test results from the alert agent;

- Inform the doctor that test results are available;

- Query the system according to search criteria determined by the doctor;

- Receive diagnosis suggestions from the Diagnostic agent.

\section{Prescription Agent (PA):}

This agent is used to improve hospital pharmacy functions, speeding the time for issuing medication order, and to improve patient safety by preventing medication errors. The agent has the capability of writing prescription, transmitting it electronically to the pharmacy and checking availability of requested medication, checking for possible drug interactions or medical conditions, which may counter-indicate the use of the medication, and keep track of patient's medications. PA usage will eliminate the prescription waiting time on part of the patients or the physicians and will decrease drug's prescription errors.

\section{Mobile Agent (MA):}

The main function of mobile agent is to search, retrieve, and deliver data according to actor's needs. Mobile agents are generated dynamically during the execution. They can reconfigure themselves dynamically based on changes of the services. Agent consists of: set of data structures to save its parameters and information which will be sent back to user; set of functions to fulfil 
users' tasks, handle events and control agents' movements, and to preserve agents' status and history record; and knowledge-based model used to fulfil its mission.

\section{Lab Agent (LA):}

Lab agent simplifies the laboratory data handling processes resulting in saving time and money. This agent should have the ability to interface with automated laboratory instruments. It's responsible for the acquisition, management and transfer of data into the patient's electronic medical record. These instruments which may come with their own software required for their operations. The agent provides real-time delivery of examination report (data and medical analysis) from laboratories that have been ordered by DA. Agent's other tasks include:

- Prioritizing the test results based on severity of patient's conditions level and informing the alert agent that test results are available.

- Inform the DA that results are ready to be sent. If the result is requested it will make it available to the DA.

\section{Radiology Agent (RA):}

- Receives orders and sends results of radiology tests performed by radiology machines.

- Communicates with image archiving system.

- Communicates with other agents such as DA, Schedule (SA), EHR, billing agents).

\section{Diagnostic Agent (DIA):}

The healthcare information systems create and store very large volumes of electronic health data related to patient services in the Case Based Database. Such huge volume of data could be used in evidence-based medicine to choose the methods of treatment and enhancing clinical decision support of the ongoing activities. The diagnosis agent has capability to access external services and suggest proper diagnosis by using Case-Based Reasoning and associated knowledge base.

\section{Mobile Device Agent (MDA):}

Agent technology support mobile medical devices with a middleware enabling them to effectively use adaptive servers within their medical network environment. Agents are particularly suitable to wireless networks as they are efficient in their use of limited network bandwidth and are able to handle data transmission in frequently disconnected wireless network. An agent can represent, communicate and work towards a medical actor's interests. Healthcare organizations are increasing their reliance on mobile links to access patient information at the point of care. The usage of mobile devices will improve the quality and reduce the cost of healthcare.

This agent is residing on the mobile device. It is responsible for gathering and maintaining information about the physical device and its owner; and is main point of contact between the mobile user and the networked resources. Actors can use mobile applications on their PDA or mobile phone to access, retrieve and view patients' profiles and to enter patients' information such as patient monitoring data, doctor's diagnosis and prescriptions in real time. Such mobile devices can be used wirelessly at different locations around the hospital or in other situations (telemedicine) where such facilities are not offered. 


\section{Home Patient Agent (HPA):}

This agent is added to the patient's premises software. It is used to monitor remote patient's conditions and transmit the data of abnormal cases to the mediator to handle routine healthcare activities and provide temporary advice and send urgent alerts to doctors in the event of an emergency.

\section{Schedule Agent (SA):}

It is used to assign the available slot of time in doctor's schedule for a patient or reschedule an appointment on the priority basis. It works in cooperation with doctor agent based on the medical consultation from patient's physician. In case of unpredicted circumstances, the agent can react flexibly to schedule a new appointment.

\section{EHR agent (EHRA):}

Electronic Health Record (EHR) is an integrated database holding patient's relevant digital information (textual, radiology images, ECGs, audio and waveform data, and other media content) generated during the care process. The EHR can be shared across the different healthcare setting as it is in digital format. Such information should be available to physicians, nurses and other medical staff.

The EHR agent provides a comprehensive set of capabilities such as:

- Real-time access to patient clinical information across healthcare systems;

- Secure access to patient's information as needed in a form convenient for the healthcare staff;

- Updating patient information in time to make fast and effective decisions.

\section{Telemedicine agent (TA):}

Telemedicine is an invaluable tool in healthcare. It provides the ability for enhancing health care delivery for patients in rural areas, reducing the travelling time for patients and health professionals, introducing training programs for medical and non-medical staff, leading to faster delivery of medical services and keeping patients satisfied.

\section{Healthcare Mediator:}

The healthcare mediator is the integrated unit which is made up of some healthcare transactions. To successfully complete healthcare transaction actors must interact. Healthcare mediator acts as the communication link between multiple agents of medical staff and patients. The mediator provides health services to local or remote patients.

\section{Methodology}

In order to validate the mechanism of the proposed architecture of intelligent distributed agent system, it was simulated on a Java-based mobile agent platform (JADE). Experimental simulation methodology has been used to justify the proposed architecture of distributed medical intelligent agent system. To observe the proposed system in real environment different simulation scenarios will be have been guided by medical actors from King Khaled Hospital. This experiment was conducted in a controlled environment to see if the proposed system is compatible with a human actor. Agents were realized using java language and were able to cooperate with each other. 
Through IA, a doctor assigns a new task by entering patient's information including patient's electronic medical record number, diagnosis standard code for the disease (ICD 10 [41]), examination and laboratory data, for example, blood test, CT, MRI and so on, to a local DA (on Figure 3). Mobile Agent MA carrying a request can travel to the intended agents (on local network or remote agents) and submit the task to them. After receiving results from these agents, MA delivers these results to DA. More than one result is possible to be worked out by multiple agents in a task. According to these results, doctors can sequentially take next step. Through interaction between involved agents, shown on the Figure 4, the system model can fulfil the doctor's tasks.

It is possible that an agent cannot be able to obtain immediately certain data for specified task because getting some results require more time. When it occurs, MA can request mediator agent to save the intermediate results to its database. When needed data are obtained, MA can travel again to mediator, request for rebuilding and joining dynamically the results and submits the data to DA to continue executing the task.

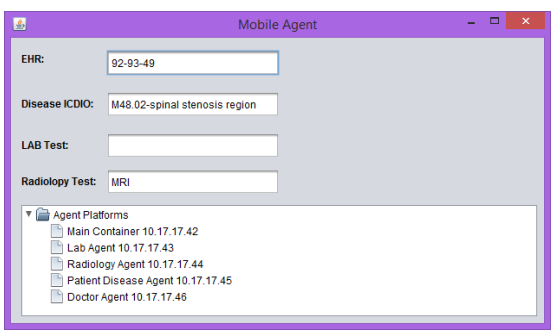

Figure 3. Interface and involved agents

Figure 3 shows a snapshot of a live simulation on the JADE agent platform where different agents are automatically started based on the doctor agent request. In this example the following agent containers are involved to perform the requested functions: lab agent, radiology agent, patient agent and doctor agent. This snapshot shows: lab agent migrated to lab server where it got patient's lab result and sent them to the Patient Database Server; the radiology agent migrated to Radiology Database Server to acquire the MRI and its report for the same patient and send them to the Patient Database Server.

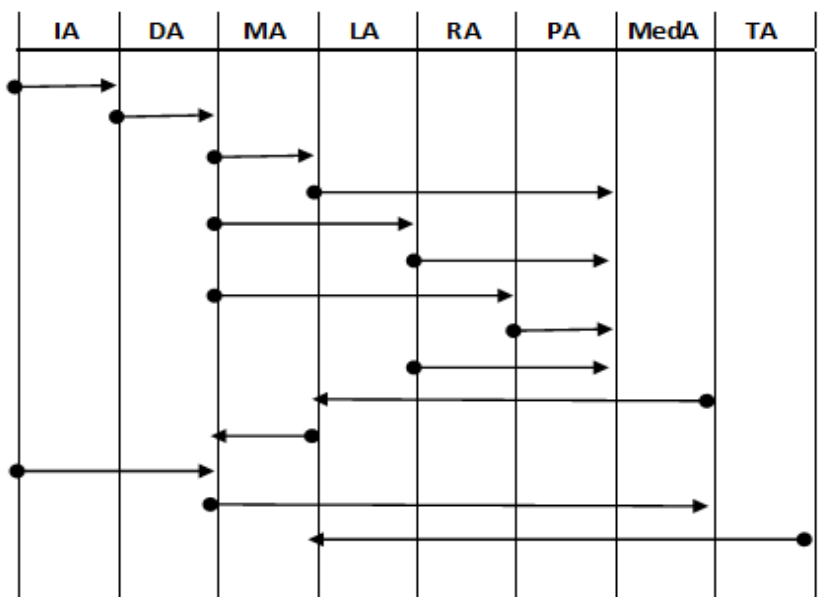

Figure 4. Agents Interactions 
Sample result for a patient as reported by the doctor agent is shown on Figure 5.

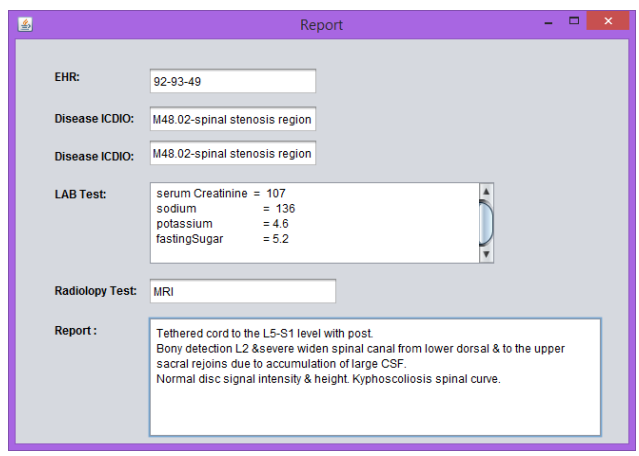

Figure 5. Result

\section{DISCUSSION}

There is a need for a great deal of coordination among different healthcare actors which occurs in a distributed way. The main challenge in this framework is tying different healthcare information systems together in execution architecture. The course of action must take into account the autonomy of the different actors. The ultimate objective of this work is to design integrated useroriented healthcare services that provide uniform access to networked healthcare information systems that supporting different contexts, and to make it easily accessible to heterogeneous healthcare actors. In addition to these services, it should provide customized functions (retrieve, share, manage, discover, etc) to the healthcare actors of specific healthcare information system. To handle these requirements, an architecture has been designed which allow the integration of information among different information infrastructures. Agent technology is employed in this framework to provide the mechanisms for integrating the content of the existing healthcare information systems and services into a uniform information system and therefore much more useful information is provided. The proposed architecture provides useful healthcare information to all healthcare community actors based on this agent-based framework. The agents could acquire, manage, and provide different types of data and information. Such technical integration is considered as a basis for organizational redesign. However, such efforts need to be handled carefully in order to avoid unforeseen problems.

The proposed approach is appropriate for adaptation of existing healthcare information systems without major modification thus reducing the implementation costs. This technique would improve the productivity of the medical staff, the quality of received information and the consistency of healthcare quality through agent's intelligence.

The major contributions of proposed framework are: designing agent-based architecture to facilitate the flow of data between various healthcare systems and enhancing the design with CBR. In the proposed architecture, multiple mobile agents work together to resolve transporting information between various distributed components, thus providing flexible dissemination architecture. The agents are able to interoperate with different subsystems systems easily, without having to be modified for each type of data platform. Agents are able to query the database servers and store the retrieved information locally over a period of time. Using the proposed approach eliminates the need for writing customized interfaces to provide translations between components. 
Implementations of the proposed architecture will allow significantly improve healthcare services. The main benefits are:

- Easy access: the connection of applications and data through agents allows the hospital staffs easy access the data of HIS and generate different records. An agent-based platform guarantees interoperability among different healthcare information systems thus ensuring the high availability and reliability of the systems.

- The mobile agent is employed to overcome the transport delay of patient's data that include multi-media content on the network.

- The interaction between humans and agents: through an interface that allows the actor to create new agents and monitor their activities in real time.

- Improvements: This system enhances integrity of information, reduces errors, and reduces information duplication.

- Reduced healthcare cost: the effective sharing and communication of data, information, and knowledge among healthcare staff is an essential factor for reducing healthcare cost.

- Healthcare policies: help the healthcare authorities to develop comprehensive healthcare policies.

- Monitoring: helps hospitals to monitor the activities and study of effectives.

\section{CONCLUSiOn\& Future WORK}

There are different types of information systems in today's large hospitals, some of these systems are complicated (in term of heterogeneity and diversity) and difficult to manage. The aim of this research paper is to devise scalable, flexible and intelligent framework architecture that use agent and CBR methodologies for enhancing highly distributed healthcare services where all data transactions are processed by agents, thus improving response time, efficiency, and availability. With the strong support of such infrastructure, the healthcare processes could be simplified and improved, and the healthcare services of different information systems of different manufacturers could be provided in an integrated manner. Using agent technology in healthcare improve its quality by providing specialized healthcare professionals (physicians, nurses and other authorized medical staff) with the right information at the right time, sharing up-to-date patient information in real time and making healthcare processes more efficient across a hospital system.

The overall contributions of this research are incorporation of agent technology and CBR in healthcare system to assist medical actors in their processes to enhance diagnostics capabilities, treatment procedures, prescriptions and recommendations; and creation of cooperative techniques for agents in a distributed medical environment. Another major contribution of this approach is the cost effectiveness of its implementation in terms of using and adapting the existing healthcare services together with information sources. In order to benefit from utilizing the proposed system, medical actors require modification of their business processes and changing their behaviour in using new technologies. This approach will improve the productivity of the medical professionals and the quality of healthcare in general.

The future work will concentrate on the security issues of this architecture. Since security is an important consideration as personal health data must be confidential. There should also be mechanisms to protect the data movement among agents and from outsiders' malicious agents. 
International Journal of Managing Information Technology (IJMIT) Vol.7, No.1, February 2015

\section{REFERENCES}

[1] Mead CN (2006). Data interchange standards in healthcare it-computable semantic interoperability: Now possible but still difficult. Do we really need a better mousetrap? J. Healthcare Inf. Manag. Vol. 20, pp 71-78.

[2] Miranda M, Machado J, Abelha A \& J Neves, (2013) "Healthcare Interoperability through a JADE Based Multi-Agent Platform”, In Intelligent Distributed Computing VI, Springer, Berlin, Germany., pp 83-88.

[3] Telecom Italia Lab, Java Agent Development Framework,http://jade.tilab.com/.

[4] Agent Oriented Software Limited (AOS), JACK Documentation, http://www.aosgrp.com/ products/jack/documentation and instruction/jack documentation.html.

[5] Acronymics Inc. AgentBuilder. http://agentbuilder.com/.

[6] IBM, Aglets, http://www.trl.ibm.com/aglets/.

[7] Khajehpour, Salari \& E. Raheleh, (2013) "Advancements and Trends in Medical Case-Based Reasoning: An Overview of Systems and System Development”, Iran J. Medical Informatics, Vol. 2, No. 4, pp 12-16.

[8] Ahmed MU, Begum S \& P Funk, (2012) "Case studies on the clinical applications using case-based reasoning", IEEE Federated Conf. on Comp Sci \& Inf Sys (FedCSIS), pp 3-10.

[9] I Bichindaritz, (2008) "Case-Based Reasoning in the Health Sciences: Why It Matters for the Health Sciences and for CBR", In Procs. 9th European Conf. on Advances in Case-Based Reasoning", Springer-Verlag, pp 1-17.

[10] Xu M \& J Shen, (2013) "Clinical Decision Support Model of Heart Disease Diagnosis Based on Bayesian Networks and Case-Based Reasoning", Proc. 19th Int. Conf. on Industrial Engineering \& Engineering Management. pp 219-225.

[11] Sharaf-El-Deen DA, Moawad IF \& ME Khalifa, (2014) "A New Hybrid Case-Based Reasoning Approach for Medical Diagnosis Systems", J. Medical Systems. pp 1-11.

[12] Gund M, Andhalkar S, Patil D \& VM Wadhai (2011) "An Intelligent Architecture for Multi-Agent Based m-Health Care System", Int. J., pp 1-5.

[13] Health Level Seven (HL7). http://www.hl7.org.

[14] Omar W \& A Taleb-Bendiab (2006). "E-Health Support Services Based on Service-Oriented Architecture", IEEE Computer Society, pp 35-41.

[15] A Ducrou, (2009) "Complete interoperability in healthcare: technical, semantic and process interoperability through ontology mapping and distributed enterprise integration techniques", Doctor of Philosophy Thesis.

[16] Kart F, Moser L. \& P Smith, (2008) “Building a Distributed E-Healthcare System Using SOA”, IEEE. Journals \& Magazines, pp 24-30.

[17] Z Xiao-Guang, (2009) "Design and Implementation of Interoperable Medical Information System Based on SOA”, ITIME '09, IEEE Int. Symp. on IT in Medicine \& Education, pp 1074-1078.

[18] Miranda, M.; Centro de Cienc. e Tecnol. da Comput., Univ. do Minho, Braga, Portugal; Pontes, G.; Abelha, A \& Neves, J. (2012) “Agent Based Interoperability in Hospital Information Systems”, Int. Conf. BioMedical Engineering and Informatics, pp 949-953.

[19] Miranda, Miguel; Salazar, Maria; Portela, Filipe; Santos, Manuel; Abelha, António; Neves, José \& José Machado, (2012) "Multi-agent Systems for HL7 Interoperability Services. Procedia Technology" 4th Conf. of Enterprise Information Systems - aligning technology, organizations and people, pp $725-733$.

[20] Nguyen M., Fuhrer P \& J Pasquier-Rocha (2009) "Enhancing E-Health Information Systems with Agent Technology”, Hindawi Publishing Corporation. Int. J. Telemedicine and Applications, pp 1-13.

[21] Subalakshmi RJ \& NCSN Iyengar, (2011) "A Small e-Health Care Information System with Agent Technology", International Conference on Computational Intelligence and Communication Networks (CICN), pp 68-72.

[22] Zheng, Shanhong; Li, Wanlong; Peng, Xinyi; Zhao, Hui \& Chunfei Zhang, (2010) "Research on Integrating the Healthcare Enterprise Based on Multi-Agent", Int. Conf. Computer, Mechatronics, Control and Electronic Engineering (CMCE), pp 493-496.

[23] Yaowen Z, Wei X \& H Yuwan, (2010) "Research on Healthcare Integrating Model of Medical Information System Based on Agent", Int. Conf. Computational and Information Sciences. pp 622625. 
International Journal of Managing Information Technology (IJMIT) Vol.7, No.1, February 2015

[24] Miller K \& G Mansingh (2013) "Towards a distributed mobile agent decision support system for optimal patient drug prescription”, 3rd Int. Conf. Innovative Computing Technology (INTECH), pp $233-238$.

[25] Cardoso L; Marins F; Portela F; Santos M; Abelha A \& J Machado, (2014) "The Next Generation of Interoperability Agents in Healthcare", Int. J. of Environmental Research and Public Health, pp 53495371.

[26] Moghaddam, Mohammad Kalmarzi; Shojafar, Mohammad; Nami, Mohammad Reza \& Hassan Rashidi, (2013) “An Efficient Multi-agent System for E-health Functionalities”, Int. J. Software Engineering and Its Applications, pp 23-34.

[27] Zarour K \& N Zarour, (2012) “A Coherent Architectural Framework for the Development of Hospital Information Systems”, Applied Medical Informatics, pp 33-41.

[28] Bromuri S, Schumacher MI, Stathis K \& J Ruiz, (2011) "Monitoring Gestational Diabetes Mellitus with Cognitive Agents and Agent Environments", IEEE/WIC/ACM Int. Conf. on Web Intelligence and Intelligent Agent Technology.

[29] Laskowski M, Demianyk BCP, Witt J, Mukhi SN, Friesen MR \& RD McLeod (2011) "Agent-Based Modeling of the Spread of Influenza-Like Illness in an Emergency Department: A Simulation Study”, IEEE Trans. Information Technology in Biomedicine, pp 877-889.

[30] Annicchiarico R, Cortés U \& C Urdiales (2008) Agent Technology and e-Health, Whitestein Series in Software Agent Technologies and Autonomic Computing. Babel, Switzerland.

[31] Minh, Tuan Nguyen; Fuhrer, Patrik \& Jacques Pasquier-Rocha, (2009) "Enhancing E-Health Information Systems with Agent Technology" International Journal of Telemedicine \& Applications, pp 1-13.

[32] Hashmi ZI, Sibte S, Abidi R \& Y Cheah, (2002) "An Intelligent Agent-based Knowledge Broker for Enterprisewide Healthcare Knowledge Procurement”, In Procs. 15th IEEE Symp. Computer Based Medical Systems (CBMS'2002), Maribor (Slovenia), pp173-178.

[33] Hadzic M, Chang E \& M Ulieru, (2006) "Soft computing agents for e-health applied to the research and control of unknown diseases", Information Sciences, Vol. 176, pp 1190-1214.

[34] Croitoru M, Hu B, Dasmahapatra S, Lewis P, Dupplaw D, Gibb A, Julia-Sape M, Vicente J, Saez C, Garcia- Gomez JM, Roset R, Estanyol F, Rafael X \& M Mier, (2007) "Conceptual Graphs Based Information Retrieval in Health Agents", Computer-Based Medical Systems, Vol. 7, No. 20- 22, pp 618-623.

[35] Sun, DFM, Chiu, DKW, Jiang N, Hu H, Zhuang Y \& H Hu, (2011) “A Multi-agent Infrastructure for Healthcare Process Improvement Using Ontology”, 8th IEEE Int. Conf. on e-Business Engineering, pp 392-396.

[36] Lee CS \& MH Wang, (2007) "Ontology-based intelligent healthcare agent and its application to respiratory waveform recognition”, Expert Systems with Applications, Vol. 33, No. 3, pp 606-619.

[37] Kifor T, Varga LZ, Salceda JV, Alvarez S, Willmott S, Miles S \& L Moreau, (2006) "Provenance in agent-mediated healthcare systems", IEEE Intelligent Systems, Vol. 21, No. 6, pp 38-46.

[38] De Meo P, Quattrone G \& D Ursino, (2011) "Integration of the HL7 Standard in a Multi-Agent System to Support Personalized Access to e-Health Services", IEEE Trans. Knowledge and Data Engineering. Vol. 23, No. 8, pp 2144-1260.

[39] Su CJ \& TW Chu, (2014) "A Mobile Multi-Agent Information System for Ubiquitous Fetal Monitoring, Int. J. Environmental Research and Public Health”, pp 600-625.

[40] Karpagam K \& A Saradha, (2014) “An Intelligent Conversation Agent for Health Care Domain”, Ictact J. Soft Computing, pp 772-776.

[41] Rinkle V \& L Akramer, (2014) "Leveraging the ICD-10 Delay", Journal of Health Care Compliance, Vol. 16, No. 5, pp 61-64.

\section{Author}

Dr. Hasan Al-Sakran currently is an Associate Professor in the Department of Management Information Systems at the King Saud University/Saudi Arabia. He has a D. Sc. degree in Information Systems Design from the George Washington University/ Washington DC. His research interests include: Agent Technology applications, E-commerce applications, Security of Information systems, Case-Based Reasoning, Software cost Estimation. 